\title{
The effects of indocyanine green and endoillumination on rabbit retina: an electroretinographic and histological study
}

\author{
A K H Kwok, T Y Y Lai, C-K Yeung, Y-S Yeung, W W Y Li, S W Chiang
}

Br J Ophthalmol 2005;89:897-900. doi: 10.1136/bjo.2004.061093

Aim: To evaluate the functional and morphological retinal toxicity associated with intravitreal injection of indocyanine green (ICG) dye in rabbit eyes during vitrectomy with endoillumination.

Methods: 20 eyes of $10 \mathrm{New}$ Zealand pigmented rabbits were used in the study. All eyes underwent pars plana vitrectomy and removal of posterior vitreous cortex under endoillumination. In one eye of each rabbit, intravitreal injection of $0.1 \mathrm{ml}$ of $2.5 \mathrm{mg} / \mathrm{ml}$ ICG was applied for 30 seconds followed by 10 minutes of endoillumination. The control eye had endoillumination only without ICG injection. Dark adapted and light adapted electroretinograms (ERGs) were performed before the surgery and 1 week after surgery for serial comparisons. Rabbits were killed 1 week after surgery and eyes were enucleated for histological examination. Results: Serial ERG comparisons showed significant reduction in the light adapted $a$-wave amplitude $(p=0.037)$ and significant delays in the dark adapted and light adapted $b$-wave latencies $(p=0.020$ and $p=0.038$, respectively) in the ICG treated eyes. Histological examinations demonstrated loss of photoreceptor outer segments with focal absence of photoreceptors in some areas in the ICG injected eyes.

Conclusions: Vitrectomy followed by intravitreal injection of $2.5 \mathrm{mg} / \mathrm{ml} \mathrm{ICG}$ for 30 seconds with endoillumination may result in retinal toxicity causing functional and morphological retinal damages in rabbit eyes. The lowest concentration of ICG should be used if necessary for intraocular use to prevent potential retinal toxicity.

$\mathrm{T}$ he use of indocyanine green (ICG) dye to stain the internal limiting membrane (ILM) for its removal during macular hole surgery has been demonstrated to improve the anatomical success rate in macular hole surgery. ${ }^{1-5}$ However, clinical studies have also reported adverse effects associated with ICG assisted ILM peeling, resulting in visual field defects and visual impairment. ${ }^{6-8}$ Several experimental studies have used cell cultures to investigate the potential retinal toxicity caused by ICG. ${ }^{9-12}$ We have previously demonstrated that ICG resulted in a concentration dependent reduction in cell viability and increased expressions of apoptosis related genes $p 53$ and bax as well as the cell cycle arrest protein p21 in human cultured retinal pigment epithelial (RPE) cells. ${ }^{10}$ Further studies have similarly shown that high concentrations of ICG caused more reductions in RPE cell viability. ${ }^{11}{ }^{12}$

Using in vitro cell culture models to study the potential ICG toxicity has the disadvantage of being unable to reproduce the functional and morphological retinal toxicity in vivo. Animal experiments were therefore performed to evaluate the in vivo retinal toxicity caused by ICG. $^{13-17}$ Since ICG related retinal toxicity may be caused by endoillumination during surgery resulting in a photosensitising effect, ${ }^{18}$ animals studies on ICG toxicity should include the use of endoillumination in the experiment. The purpose of our study is to investigate the retinal toxicity in rabbit eyes after the application of ICG during vitrectomy with endoillumination through retinal electroretinography and histology.

\section{MATERIALS AND METHODS \\ Animals}

Twenty eyes of 10 New Zealand pigmented rabbits were used in the study. All animals were treated in accordance with the ARVO statement for the use of animals in ophthalmic and vision research and the protocol was approved by the committee on animal research of The Chinese University of Hong Kong.

\section{Surgical procedure}

The rabbits were anaesthetised with an intramuscular injection of $15 \mathrm{mg} / \mathrm{kg}$ xylazine hydrochloride, $50 \mathrm{mg} / \mathrm{kg}$ ketamine hydrochloride, and $60 \mathrm{mg} / \mathrm{kg}$ sodium pentobarbital. Both pupils were dilated with $0.5 \%$ tropicamide and $0.5 \%$ phenylephrine eye drops. Following disinfection with 5\% povidone-iodine and draping of the surgical field, standard three port pars plana vitrectomy with removal of the posterior vitreous cortex under endoillumination was performed in all eyes. In the eyes receiving ICG injection, $0.1 \mathrm{ml}$ of $2.5 \mathrm{mg} / \mathrm{ml}$ ICG solution was applied gently over the optic disc with the infusion temporarily stopped. ICG was prepared by diluting $25 \mathrm{mg}$ of commercially available stock ICG powder with the $10 \mathrm{ml}$ solution provided (Diagnogreen Injection, Daiichi Pharmaceutical, Tokyo, Japan). After 30 seconds, the intravitreal ICG solution was aspirated out with resumption of the infusion solution. Endoillumination was then applied for 10 minutes in eyes with or without ICG injection using a surgical light pipe connected to a tungsten halogen light source (Premiere, Storz Inc, USA). The light pipe was put in the mid-vitreous in order to provide an even illumination to the posterior pole. The sclerotomies and conjunctival wounds were then closed and subconjunctival injection of $5 \mathrm{mg}$ gentamicin was given. Postoperatively, all rabbits received tobramycin ointment twice daily for 1 week.

\section{Electroretinogram recording}

Full field electroretinogram (ERG) was performed on all eyes before surgery and 1 week after surgery. All eyes were dilated with $0.5 \%$ phenylephrine and $0.5 \%$ tropicamide drops and dark adapted for 30 minutes before recordings. Stimuli were delivered by a xenon strobe light mounted on a Ganzfeld dome. Responses were recorded with a computerised recording unit with amplifier filtered at frequencies from $0.5 \mathrm{~Hz}$ to $1 \mathrm{kHz}$ (Nicolet Biomedicals, Madison, WI, USA). The rabbits were positioned in front of the dome and Burian-Allen

Abbreviations: ERG, electroretinogram; ICG, indocyanine green; ILM, internal limiting membrane; RPE, retinal pigment epithelium 
A
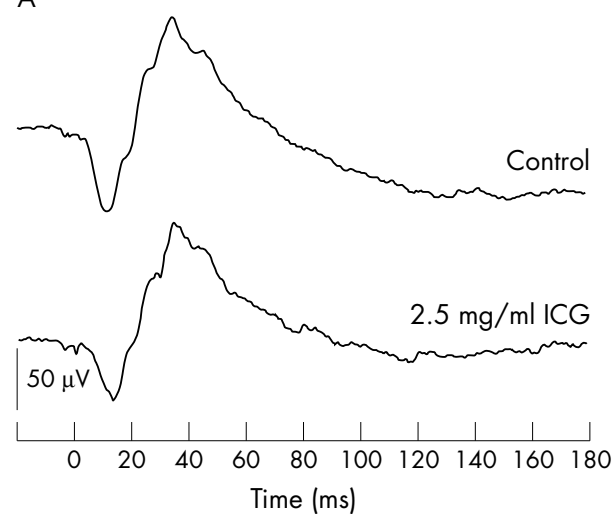

B
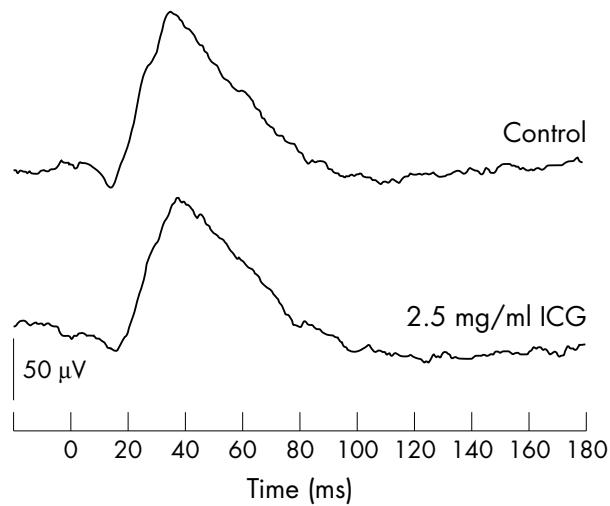

Figure 1 (A) The dark adapted ERG waveforms and (B) light adapted ERG waveforms of $2.5 \mathrm{mg} / \mathrm{ml}$ ICG treated and control rabbit eyes.

bipolar contact lens electrodes (Hansen Laboratory, Iowa City, IA, USA) and grounding earclip electrodes were used for recordings. Dark adapted ERG was performed with a white stimulus and a total of eight responses were averaged and light adapted ERG was recorded after light adapting the rabbit for 5 minutes. The responses to 20 bright white flashes at $1 \mathrm{~Hz}$ were recorded. The average of the control eyes was taken as $100 \%$ and the average of the treated eyes was calculated as the relative percentage of the control eye. ${ }^{19}$ To avoid diurnal variation in ERG responses, all ERGs were performed at the same time of day. ERG were analysed by measuring the latency and amplitude of the a-waves and b-waves. The preoperative ERGs and the ERG performed 1 week after surgery were compared using the paired two tailed $t$ test. A p value of $\geqslant 0.05$ was considered statistically significant.

\section{Specimen preparation for light microscopy}

Rabbits were killed by an intravenous injection of $120 \mathrm{mg} / \mathrm{kg}$ sodium pentobarbital 1 week after surgery and the eyes were enucleated. All eyes were sectioned in half and fixed in $4^{\circ} \mathrm{C}$ mixture of $2.5 \%$ glutaraldehyde and $4 \%$ paraformaldehyde in $0.1 \mathrm{M}$ phosphate buffer at $\mathrm{pH}$ 7.4. After fixation, the specimens were stained en bloc with lead citrate, washed three times in $0.1 \mathrm{M}$ phosphate buffer, and serially dehydrated with ethyl alcohol. The specimens were embedded and stained with haematoxylin and eosin for light microscopy.

\section{RESULTS}

\section{Electroretinography analysis}

Figure 1 shows the dark adapted and light adapted ERG waveforms of the rabbits at 1 week after surgery. There was
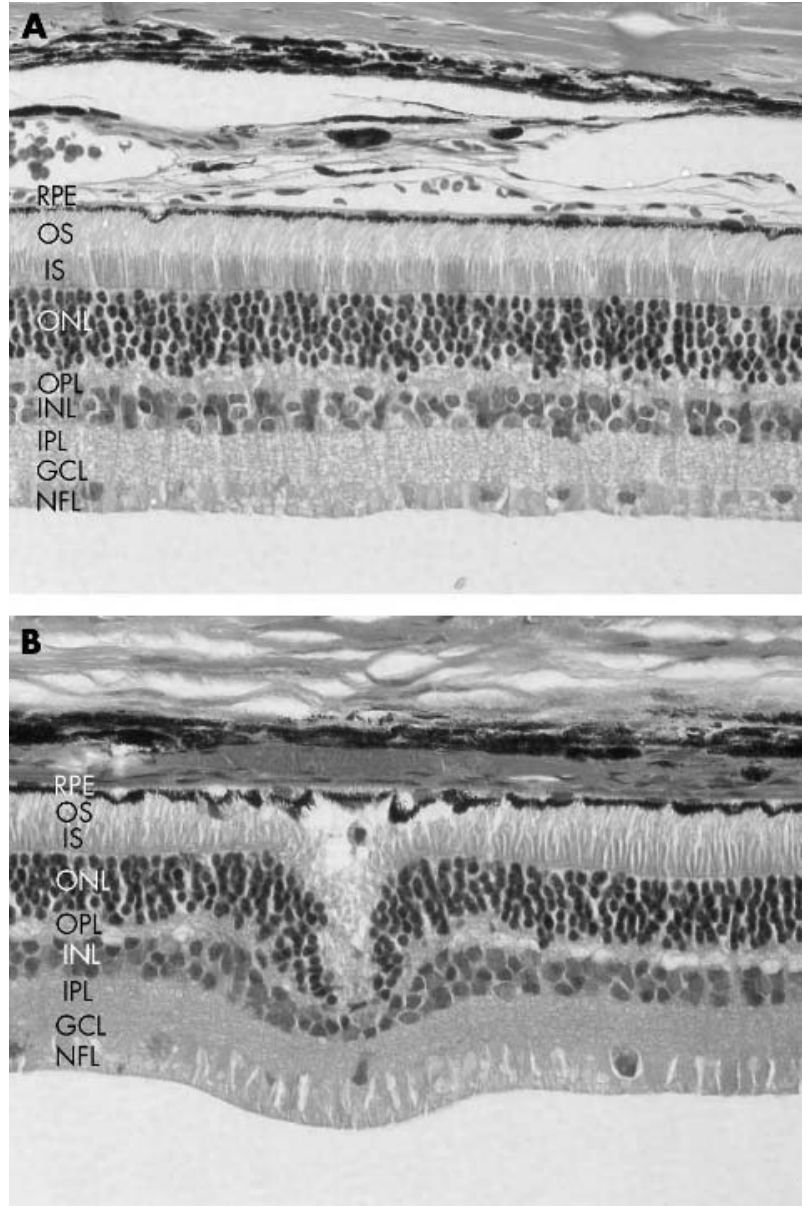

Figure 2 (A) Light microscopy of eyes without ICG injection showing preservation of retinal morphology in all layers. (B) Light microscopy of eyes treated with $2.5 \mathrm{mg} / \mathrm{ml}$ ICG showing loss of the outer segments of the photoreceptors, focal absence of photoreceptors, and localised areas of RPE irregularities. Focal areas of cellular oedema were also seen in the outer nuclear and inner nuclear layers. (RPE, retinal pigment epithelium; OS, outer segment; IS, inner segment; ONL, outer nuclear layer; OPL, outer plexiform layer; INL, inner nuclear layer; IPL, inner plexiform layer; $\mathrm{GCL}$, ganglion cell layer; NFL, nerve fibre layer. Haematoxylin and eosin stain, original magnification $\times 400$.)

no difference in the shape of the waveforms recorded in the eyes treated with ICG compared with control eyes. Table 1 demonstrates the ERG results expressed as relative ratios of the ICG treated eyes compared with control eyes. For the dark adapted ERG, no significant changes were observed in the ratios of the a-wave latency, a-wave amplitude, and b-wave amplitude compared with the baseline ERG. However, there was a significant delay in the dark adapted b-wave ERG latency for the ICG treated eyes compared with control after surgery (two tailed $t$ test, $\mathrm{p}=0.020$ ). For the light adapted ERG, there was a significant reduction in the a-wave amplitude and significant increase in the b-wave latency for the ICG treated eyes after surgery (two tailed $t$ test, $p=0.037$ and $p=0.038$ respectively). No significant changes in the a-wave latency and the b-wave amplitude after surgery were observed.

\section{Light microscopic analysis}

In the control eyes without ICG injection, the normal structure of the retina was preserved (fig 2A). However, in eyes that were treated with ICG, localised areas of RPE irregularities could be seen. There were also losses of the outer segments of the photoreceptors together with focal 
Table 1 Results of the dark adapted and light adapted ERG

\begin{tabular}{llll}
\hline ERG component & $\begin{array}{l}\text { Baseline } \\
\text { (mean (SEM)) }\end{array}$ & $\begin{array}{l}\text { 1 week after surgery } \\
\text { (mean (SEM)) }\end{array}$ & p Value \\
\hline $\begin{array}{llll}\text { Dark adapted } \\
\text { a-wave latency }\end{array}$ & $1.05(0.01)$ & $1.01(0.03)$ & 0.18 \\
a-wave amplitude & $1.20(0.23)$ & $1.47(0.37)$ & 0.54 \\
b-wave latency & $0.98(0.02)$ & $1.05(0.01)$ & $0.020^{*}$ \\
b-wave amplitude & $0.93(0.03)$ & $1.00(0.04)$ & 0.11 \\
$\begin{array}{l}\text { Light adapted } \\
\text { a-wave latency } \\
\text { a-wave amplitude }\end{array}$ & $0.99(0.03)$ & $0.98(0.04)$ & 0.87 \\
b-wave latency & $1.35(0.34)$ & $0.69(0.10)$ & $0.037^{*}$ \\
b-wave amplitude & $0.97(0.02)$ & $1.05(0.03)$ & $0.038^{*}$ \\
\hline *Statistically significant, two tailed $t$ test $(\mathrm{n}=20)$. & $0.96(0.10)$ & 0.40 \\
\hline
\end{tabular}

absence of photoreceptors in some areas (fig 2B). In the outer nuclear and inner nuclear layers, focal areas of cellular oedema with irregularities were detected.

\section{DISCUSSION}

Several animal studies have evaluated the potential retinal toxicity of subretinal and intravitreal ICG injections and most studies showed there is a concentration dependent retinal toxicity associated with ICG. ${ }^{13-17}$ Maia et al demonstrated that ERG and histology were normal 1 week after intravitreal injection of $0.5 \mathrm{mg} / \mathrm{ml}$ ICG in rabbit eyes, whereas injections of higher concentrations of $5 \mathrm{mg} / \mathrm{ml}$ and $25 \mathrm{mg} / \mathrm{ml} \mathrm{ICG}$ resulted in dark adapted ERG abnormalities. ${ }^{17}$ Histological examination also revealed morphological changes in eyes injected with these ICG concentrations. In another experiment, Enaida et al performed gas vitrectomy using $100 \% \mathrm{SF}_{6}$ gas followed by intravitreal ICG injection to evaluate the morphological and functional damage caused by ICG in rat eyes, which showed that ICG injection resulted in functional changes detected on dark adapted b-wave even at a low dose of $0.25 \mathrm{mg} / \mathrm{ml} .{ }^{16}$ However, since no endoillumination was used in these experiments, the retinal toxicity caused by the potential photosensitising effects of ICG may not be adequately assessed. Furthermore, the injection of ICG in these experiments was not aspirated from the vitreous cavity and the retained ICG might have therefore caused prolonged contact with the retina resulting in toxicity. In the experiment by Maia et al, ${ }^{17}$ the concentrations of $5 \mathrm{mg} / \mathrm{ml}$ and $25 \mathrm{mg} / \mathrm{ml}$ ICG used may also be too high, as the maximum concentration of ICG that is commonly used for macular surgery is $2.5 \mathrm{mg} / \mathrm{ml}$. We therefore decided to investigate the effects of intravitreal injection of $2.5 \mathrm{mg} / \mathrm{ml}$ ICG for a short duration of 30 seconds after pars plana vitrectomy, followed by endoillumination in order to reproduce the clinical situation more realistically.

This study demonstrated that intravitreal application of $2.5 \mathrm{mg} / \mathrm{ml}$ ICG with endoillumination resulted in significant reduction in the light adapted a-wave amplitude and significant delays in both dark adapted and light adapted b-wave latency compared with control eyes. Morphological changes with loss of photoreceptor outer segments were also seen in ICG treated eyes upon histological examinations. Both functional and morphological toxicity occurred despite having an ICG contact time of only 30 seconds. Since the light adapted a-wave ERG response mainly originates from the cone photoreceptors, the results suggest that cone photoreceptors may be more susceptible to ICG toxicity. The observed delays in b-wave latencies also suggest that ICG may result in toxicity to the on-bipolar cells or the Müller cells. Although, it is unclear whether the ERG changes are reversible, these changes may be transient as Maia et al showed that some of the ERG changes were no longer significant 1 month after intravitreal ICG injection. ${ }^{17}$

These results should be interpreted with caution as despite the replication of the surgical method with the use of endoillumination, the experiments still could not mimic the ICG use in humans during vitrectomy as the vitreous cavity of rabbit eyes is smaller than that of humans, which means a higher concentration of ICG might accumulate in the rabbit eye. ${ }^{17}$ This study also has the limitation of investigating only one concentration of ICG and thus is unable to demonstrate the concentration dependent toxicity caused by ICG. Another limitation is the relatively short follow up duration with only one time interval studied after surgery. None the less, in view of the potential retinal toxicity, the minimal concentration of ICG should be used during surgery if required. Diluting the ICG solution with balanced salt solution for iso-osmolarity or with dextrose for preparing infracyanine green solution can

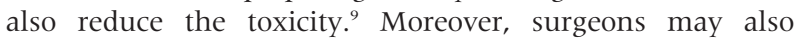
consider using lower light levels, shorter light exposure, as well as using different wavelengths of light in order to avoid potential clinical toxicity.

\section{Authors' affiliations}

A K H Kwok, T Y Y Lai, C-K Yeung, Y-S Yeung, W W Y Li, S W Chiang, Department of Ophthalmology and Visual Sciences, The Chinese University of Hong Kong, Hong Kong Eye Hospital, Hong Kong, People's Republic of China

A K H Kwok, Department of Ophthalmology, Hong Kong Sanatorium and Hospital, Hong Kong, People's Republic of China

Correspondence to: Dr Timothy Y Y Lai, Department of Ophthalmology and Visual Sciences, The Chinese University of Hong Kong, Hong Kong Eye Hospital, 147K Argyle Street, Kowloon, Hong Kong;

tyylai@netvigator.com

Accepted for publication 1 January 2005

\section{REFERENCES}

1 Kadonosono $\mathrm{K}$, Itoh N, Uchio $\mathrm{E}$, et al. Staining of internal limiting membrane in macular hole surgery. Arch Ophthalmol 2000;118:1116-18.

2 Da Mata AP, Burk SE, Riemann CD, et al. Indocyanine green-assisted peeling of the retinal internal limiting membrane during vitrectomy surgery for macular hole repair. Ophthalmology 2001;108:1187-92.

3 Kwok AK, Lai TY, Man-Chan W, et al. Indocyanine green staining and removal of retinal internal limiting membrane in stage 3 or 4 macular hole surgery. Br J Ophthalmol 2003;87:71-4.

4 Kwok AK, Lai TY, Yuen KS, et al. Macular hole surgery with or without indocyanine green stained internal limiting membrane peeling. Clin Experiment Ophthalmol 2003;31:470-5.

5 Slaughter K, Lee IL. Macular hole surgery with and without indocyanine green assistance. Eye 2004;18:376-8.

6 Haritoglou C, Gandorfer A, Gass CA, et al. Indocyanine green-assisted peeling of the internal limiting membrane in macular hole surgery affects visual outcome: a clinicopathologic correlation. Am J Ophthalmol $2002 ; 134: 836-41$. 
7 Uemura A, Kanda S, Sakamoto Y, et al. Visual field defects after unevenfful vitrectomy for epiretinal membrane with indocyanine greenassisted internal limiting membrane peeling. Am J Ophthalmol 2003;136:252-7.

8 Ando $\mathrm{F}$ Sasano $\mathrm{K}$, Ohba $\mathrm{N}$ et al Anatomic and visual outcomes after indocyanine green-assisted peeling of retinal internal limiting membrane in idiopathic macular hole surgery. Am J Ophthalmol 2004;137:609-14.

9 Stalmans $\mathbf{P}$, Van Aken EH, Veckeneer $M$, et al. Toxic effect of indocyanine green on retinal pigment epithelium related to osmotic effects of the solvent. Am J Ophthalmol 2002;134:282-5.

10 Yam HF, Kwok AK, Chan KP, et al. Effect of indocyanine green and illumination on gene expression in human retinal pigment epithelial cells. Invest Ophthalmol Vis Sci 2003:44:370-7.

11 Ho JD, Tsai RJ, Chen SN, et al. Cytotoxicity of indocyanine green on retinal pigment epithelium: implications for macular hole surgery. Arch Ophthalmol 2003;121:1423-9.

12 Rezai KA, Farrokh-Siar L, Ernest JT, et al. Indocyanine green induces apoptosis in human retinal pigment epithelial cells. Am J Ophthalmol 2004; 137:931-3.
13 Lee JE, Yoon TJ, Oum BS, et al. Toxicity of indocyanine green injected into the subretinal space: subretinal toxicity of indocyanine green. Retina 2003;23:675-81.

14 Kawaji T, Hirata A, Inomata Y, et al. Morphological damage in rabbit retina caused by subretinal injection of indocyanine green. Graefes Arch Clin Exp Ophthalmol 2004;242:158-64.

15 Maia $M$, Kellner L, de Juan $E \mathrm{Jr}$, et al. Effects of indocyanine green injection on the retinal surface and into the subretinal space in rabbits. Retina 2004:24:80-91.

16 Enaida H, Sakamoto T, Hisatomi T, et al. Morphological and functional damage of the retina caused by intravitreous indocyanine green in rat eyes. Graefes Arch Clin Exp Ophthalmol 2002;240:209-13.

17 Maia M, Margalit E, Lakhanpal R, et al. Effects of intravitreal indocyanine green injection in rabbits. Retina 2004:24:69-79.

18 Haritoglou C, Gandorfer A, Schaumberger M, et al. Light-absorbing properties and osmolarity of indocyanine-green depending on concentration and solvent medium. Invest Ophthalmol Vis Sci 2003;44:2722-9.

19 Jaffe GJ, Yang CH, Guo H, et al. Safety and pharmacokinetics of an intraocular fluocinolone acetonide sustained delivery device. Invest Ophthalmol Vis Sci 2000;41:3569-75. 